\title{
Genetic polymorphisms of glutathione S-transferase M1 and T1, and evaluation of oxidative stress in patients with non-small cell lung cancer
}

\author{
Hongyan Zhang ${ }^{1}$, Xuwei Wu' ${ }^{1}$, Yi Xiao ${ }^{1}$, Mei Chen ${ }^{1}$, Zhidong $\mathrm{Li}^{1}$, Xing Wei ${ }^{1}$ and Kaifa Tang ${ }^{2^{*}}$
}

\begin{abstract}
Background: Our objective is to investigate the genetic polymorphisms of the glutathione S-transferase M1 and T1 genes (GSTM1 and GSTT1) and evaluate oxidative damage in patients with non-small lung cancer (N-SCLC).

Methods: One hundred and ten patients with N-SCLC and 100 controls are included in this case-control study. Multiplex polymerase chain reaction (PCR) analyses were used to identify the genotypes. The activities of malondialdehyde (MDA) and nitric oxide (NO) and total antioxidant capacity (T-AOC) were detected by spectroscopic analysis.

Results: The frequencies of the GSTM1, T1, and GSTM1/T1 null genotypes in the patient group were significantly higher than that in the control group $(\mathrm{OR}=2.071, P=0.009 ; \mathrm{OR}=1.900, P=0.024 ; \mathrm{OR}=3.258, P=0.003)$. The activities of MDA and NO were significantly higher in the patient group than that in the control group $(P<0.001)$, and T-AOC was significantly lower in patient group than that in control group $(P<0.001)$. The activities of MDA, and NO were higher but the T-AOC was lower in patients with the GSTM1, T1 and M1/T1 null genotypes than those in patients with GSTM1, $\mathrm{T} 1$ and $\mathrm{M} 1 / \mathrm{T} 1$ present genotypes $(P<0.001)$.

Conclusions: Our results suggest that oxidative damage may be play a important role in patients with N-SCLC, and that GSTM1 and GSTT1 null genotypes may predispose the cells of patients with N-SCLC to increased oxidative damage.
\end{abstract}

Keywords: Glutathione S-transferases, Polymorphism, Oxidative stress, Non-small lung cancer

\section{Background}

Lung cancer remains the leading cause of cancer death in the United States and in European countries, as well as in Asian countries, mainly due to late presentation [1]. Among various histological types of lung cancers, non-small cell lung cancer (N-SCLC) accounts for approximately $80 \%$. However, knowledge on early detection, therapeutic progress, and the prognosis of N-SCLC patients remain poor [2].

Lung is a primary organ with large surface area that is directly exposed to ambient air, and therefore higher oxygen tensions, and is known to regulate reactive oxygen species

\footnotetext{
*Correspondence: doc.tangkf@hotmail.com

${ }^{2}$ Affiliated Hospital of Guiyang Medical College, No. 9 Beijing Road, Guiyang 550004, China

Full list of author information is available at the end of the article
}

(ROS) production [3]. The lung cells experience enhanced oxidative stress (OS) by exogenous free radical-generating environmental irritants and pollutants, including oxidants such as cigarette smoke, ozone, and endogenous factors like inflammation and activation of inflammatory cells [4]. Previous study has shown that oxidative stress and free radicals have been associated with an increased risk of various cancers [5]. Others studies have suggested that exposure to OS leads to single and clustered damage to cellular DNA and is involved in mutations and genomic instability, which eventually results in malignant transformation [6,7]. Ito et a1. demonstrated that low antioxidant capacity (AOC) in those who never smoked but that have N-SCLC may have contributed to excessive oxidative DNA damage in the lung 
tissues [8]. Peddireddy et al. showed that an evidencebased increased rate of oxidative stress plays a role in the pathogenesis of N-SCLC because a failure in the oxidant/antioxidant balance favors lipid peroxidation and DNA damage [9].

Glutathione S-transferases (GST) are a family of phase II enzymes, which uses reduced glutathione in a conjugation and in a reduction reaction to eliminate many different toxic electrophiles and products of oxidative stress [10,11]. Glutathione S-transferases (GSTM1) are able to detoxify benzopyrene diolepoxide, whereas glutathione S-transferases T1 (GSTT1) can conjugate oxidized lipids and halogenated compounds [12]. GSTM1 and GSTT1 are expressed in lung tissues [13]. GSTM1 (1p13) and GSTT1 (22q 11.2) genes, encoding for a $\mu$-GST isoenzyme and a $\theta$-GST isoenzyme, respectively, can be deleted, thereby causing a lack of the respective enzyme function [14]. Some previous studies suggested that GSTM1 and GSTT1 null genotypes may be associated with increased susceptibility to lung cancer $[15,16]$, but other studies have shown that there no association between GSTM1 and T1 null genotypes for lung cancer risk $[17,18]$.

However, few reports have investigated the relationship between GSTM1 and T1 genotypes and the level of oxidative stress (OS) in patients with N-SCLC. In this study, our intention is to determine the genotypic frequencies of the GSTM1 and T1 polymorphisms, and to evaluate the OS in patients with N-SCLC from Yunnan Province of China. The genotypes of GSTM1 and T1 and oxidative biochemical markers such as malondialdehyde (MDA), nitric oxide (NO) and total antioxidant capacity (T-AOC) were detected in each sample.

\section{Methods}

\section{Participants}

This case-control study consisted of 110 patients with primary N-SCLC and 100 healthy controls from Yunnan Province of China. The mean age, sex, performance status (PS), and smoking habits are shown in Table 1. N-SCLC was histologically confirmed in all patients, and all $\mathrm{N}$-SCLC patients were evaluated and staged at their first visit according to medical history, physical examination including PS by Eastern Cooperative Oncology Group stage, complete blood count, serum biochemistry analyses, chest X-ray, and computed tomography scans. The controls were selected from a pool of healthy volunteers who visited the general health checkup center during the same period. A detailed questionnaire was completed for each case and control by a trained interviewer. All controls had no known medical illness or hereditary disorders and were taking no medications. The protocol was approved by the Ethics Committee of the Affiliated Yan'an Hospital of Kunming Medical University.
Table 1 The descriptive statistics for the demographic and clinical characteristics of all participants

\begin{tabular}{llll}
\hline & $\begin{array}{l}\text { N-SCLC } \\
(\mathbf{n = 1 1 0})\end{array}$ & $\begin{array}{l}\text { Controls } \\
(\mathbf{n}=\mathbf{1 0 0})\end{array}$ & P value \\
\hline Mean age \pm SD (Age range) & $60.18 \pm 8.64$ & $59.23 \pm 11.12$ & 0.493 \\
Sex & $16(14.55)$ & $13(13.00)$ & - \\
Female (\%) & $94(85.45)$ & $87(87.00)$ & 0.746 \\
Male (\%) & & & \\
Smoking history ${ }^{\mathrm{a}}$ & $50.38 \pm 20.25$ & $48.02 \pm 20.76$ & 0.405 \\
Mean pack-years \pm SD & & & - \\
Performance status & & - & - \\
0 to 1 (\%) & $83(75.45)$ & - & \\
2 to 4 (\%) & $27(24.55)$ & & - \\
\hline
\end{tabular}

$N$-SCLC, non-small cell lung cancer; $S D$, standard deviation.

${ }^{\text {a S} m o k i n g ~ h i s t o r y ~ i n ~ p a c k-y e a r s . ~}$

b Eastern Cooperative Oncology Group performance status.

\section{Glutathione S-transferase gene polymorphisms}

An AxyPrep TM Genomic DNA Miniprep Kit (Axygen Biosciences, Union City, CA, USA) was used to isolate genomic DNA from peripheral blood samples. The GSTM1 and GSTT1 genotypes were identified by multiplex polymerase chain reaction (PCR) using published primer sequences as follows: GSTM1 gene, 5'-GAA CTC CCT GAA AAG CTA AAG C-3' (forward) and 5'-GTT GGG CTC AAA TAT ACG GTG G-3' (reverse); GSTT1 gene, 5'-TTC CTT ACT GGT CCT CAC ATC TC-3' (forward) and $5^{\prime}$-TCA CCG GAT CAT GGC CAG CA-3' (reverse);

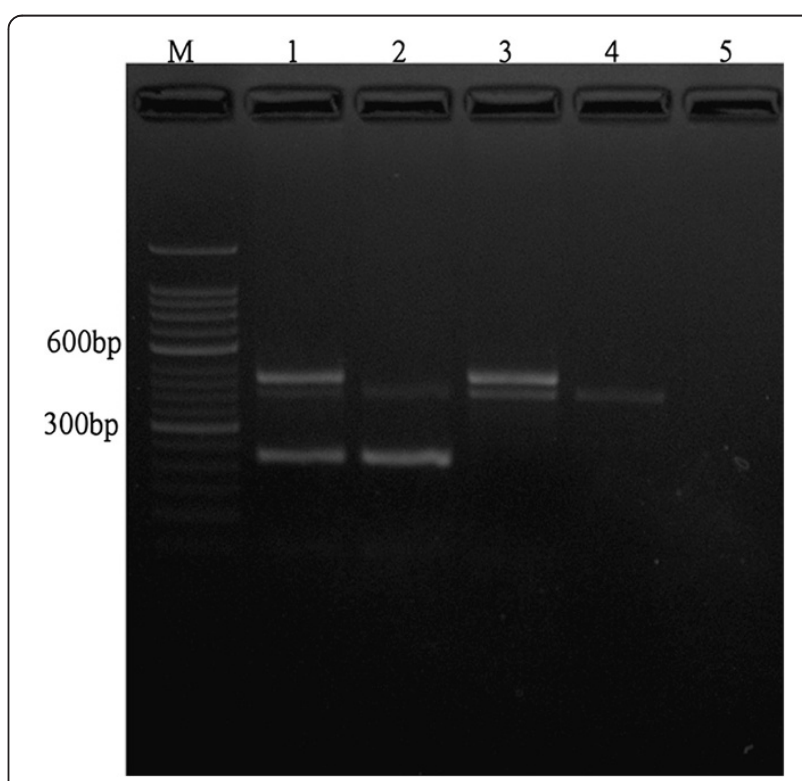

Figure $1 \mathrm{~A}$ representative image of multiplex polymerase chain reaction (PCR) analysis of glutathione S-transferase $\mathrm{M} 1$ and $\mathrm{T} 1$ (GSTM1/T1), and $\beta$-actin gene polymorphisms. Lane $M, 50-b p$ DNA marker; Lane 1, GSTM1/T1 (+/+) genotype; Lane 2, GSTM1/T1 (+/-) genotype; Lane 3, GSTM1/T1 (-/+) genotype; Lane 4, GSTM1/T1 (-/-) genotype and Lane 5, negative control. 
and a 400-bp fragment for the $\beta$-actin gene $5^{\prime}$-ACT CCC CAT CCC AAG ACC-3' (forward) and 5'-CCT TAA TGT CAC GCA CGA T-3' (reverse) was used as an internal control for DNA amplification [19] (Figure 1).

\section{Measurement of malondialdehyde, total antioxidant capability and nitric oxide}

The contents of malondialdehyde (MDA), total antioxidant capability (T-AOC) and nitric oxide (NO) in plasma were assayed using colorimetric methods with a spectrophotometer (Biomate 5, Thermo Electron Corporation, Rochester, NY, USA). The assays were conducted using the assay kits purchased from Nanjing Jiancheng Institute of Bioengineering (Nanjing, China) and used according to the manufacturer's instructions.

\section{Statistical analysis}

The $x^{2}$ test was used to compare sex and cigarette smoke between patients and controls. All data are expressed as the mean \pm standard deviation (SD). Age, smoking index expressed as pack-years (number of cigarettes smoked per day $\times$ number of years smoked/20) were compared using the unpaired Student's $t$ test. The differences in the frequencies of the GST genotypes between groups were analyzed using the $x^{2}$ test, and the odds ratios (OR) with $95 \%$ confidence intervals (CI) are reported. The difference between the two groups was analyzed by unpaired $t$ test with two-tailed values, and $P<0.05$ was considered statistically significant. All analyses were performed using SPSS software version 16.0 (SPSS, Inc., Chicago, IL, USA).

\section{Results and discussion}

No statistically significant differences were found between patients and controls with respect to age, sex and smoking history. The frequencies of the GSTM1 and T1 genotypes in cases and controls are shown in Table 2. The frequency of the GSTM1 null genotype was $42.0 \%$ in the control group and $60.0 \%$ in the patient group $(\mathrm{OR}=2.071$; CI $95 \%, 1.194$ to $3.593 ; P=0.009)$, and $34.0 \%$ and $78.0 \%(\mathrm{OR}=4.132$; CI $95 \%, 1.957$ to 8.724 ; $P<0.001)$ in only GSTT1 null genotype cases, and $51.1 \%$ and $52.9 \%(\mathrm{OR}=0.719$; CI $95 \%, 0.298$ to $1.734 ; P=0.462)$ in only GSTT1 present genotype cases. The frequency of the GSTT1 null genotype was $53.0 \%$ in the control group and $69.2 \%$ in the patient group $(\mathrm{OR}=1.900$; CI 95\%, 1.084

Table 2 The distribution of glutathione S-transferase (GST) M1 and T1 genotypes in study groups

\begin{tabular}{|c|c|c|c|c|c|}
\hline Group & $\mathrm{N}-\operatorname{SCLC}(\%)(n=110)$ & Control $(\%)(n=100)$ & $x^{2}$ & $P$ value & OR (CI 95\%) \\
\hline GSTT1 total & $(n=110)$ & $(n=100)$ & & & \\
\hline GSTM1(+) & $44(40.0)$ & $58(58.0)$ & - & - & - \\
\hline$(-)$ & $66(60.0)$ & $42(42.0)$ & 6.794 & 0.009 & 2.071 (1.194 to 3.593 ) \\
\hline GSTT1(-) & $(n=75)$ & $(n=53)$ & & & \\
\hline GSTM1(+) & $24(32.0)$ & $35(66.0)$ & - & - & - \\
\hline$(-)$ & $51(78.0)$ & $18(34.0)$ & 14.480 & $<0.001$ & $4.132(1.957$ to 8.724$)$ \\
\hline GSTT1(+) & $(n=35)$ & $(n=47)$ & & & \\
\hline GSTM1(+) & $20(57.1)$ & $23(48.9)$ & - & - & - \\
\hline$(-)$ & $15(52.9)$ & $24(51.1)$ & 0.542 & 0.462 & 0.719 (0.298 to 1.734$)$ \\
\hline GSTM1 total & $(n=110)$ & $(n=100)$ & & & \\
\hline GSTT1(+) & $35(31.8)$ & $47(47.0)$ & - & - & - \\
\hline$(-)$ & $75(69.2)$ & $53(53.0)$ & 5.073 & 0.024 & $1.900(1.084-3.331)$ \\
\hline GSTM1(-) & $(n=66)$ & $(n=42)$ & & & \\
\hline GSTT1(+) & $15(22.7)$ & $24(57.1)$ & - & - & - \\
\hline$(-)$ & $51(77.3)$ & $18(52.9)$ & 13.177 & $<0.001$ & $4.533(1.958-10.496)$ \\
\hline GSTM1(+) & $(n=44)$ & $(n=58)$ & & & \\
\hline GSTT1(+) & $20(45.5)$ & $23(39.7)$ & - & - & - \\
\hline$(-)$ & $24(54.5)$ & $35(60.3)$ & 2.233 & 0.135 & $1.826(0.826-4.036)$ \\
\hline \multicolumn{6}{|l|}{ GSTM1/T1 } \\
\hline$(+/+)$ & $20(18.2)$ & $23(23.0)$ & - & - & - \\
\hline$(+/-)$ & $24(21.8)$ & $35(35.0)$ & 0.345 & 0.557 & $0.789(0.357-1.743)$ \\
\hline$(-/+)$ & 15 (13.6) & $24(24.0)$ & 0.542 & 0.462 & $0.719(0.298-1.734)$ \\
\hline$(-/-)$ & $51(46.4)$ & $18(18.0)$ & 8.571 & 0.003 & 3.258 (1.457-7.287) \\
\hline
\end{tabular}

+ , present genotype; -, null genotype; $\mathrm{OR}$, odds ratio; $\mathrm{Cl}$, confidence intervals. 
to $3.331 ; P=0.024)$, and $52.9 \%$ and $77.3 \%(\mathrm{OR}=4.533$; $\mathrm{CI}$ $95 \%, 1.958$ to $10.496 ; P<0.001)$ in only GSTM1 null genotype cases, and $60.3 \%$ and $54.5 \%$ (OR $=1.826$; CI 95\%, 0.826 to $4.036 ; P=0.135$ ) in only GSTM1 present genotype cases. The frequency of the GSTM1/T1 null genotype was $18.0 \%$ in the control group and $46.4 \%$ in the patient $(\mathrm{OR}=3.258$; $\mathrm{CI} 95 \%, 1.457$ to $7.287 ; P=0.003)$. There were significant differences between the control group and patient group with respect to the frequencies of the GSTM1 and T1 genotypes in this study.

Glutathione S-transferase M1 and T1 polymorphisms with respect to MDA, NO, and T-AOC in the plasma are displayed in Table 3. The data of MDA, NO and T-AOC were distributed in a nearly normal fashion. The activity of MDA and NO were significantly higher in the patient group than in the control group $(P<0.001)$, and T-AOC was significantly lower in the patient group than in the control group $(P<0.001)$. Furthermore, the activities of MDA and NO in the GSTM1, T1 and M1/T1 null genotype groups were statistically significantly higher than that with the GSTM1, T1 and M1/T1 present genotypes $(P<0.001)$. However, the levels of T-AOC in the GSTM1, T1 and M1/T1 null genotypes groups were statistically significantly lower than that with the GSTM1, T1 and M1/T1 present genotypes $(P<0.001)$. In addition, we found that the GSTM1 and GSTT1 genotypes also affect the levels of MDA, NO and T-AOC in the control group. The levels of MDA and NO in the GSTM1 and GSTT1 null genotype groups were higher than that in present genotype group, and T-AOC was lower in present genotype group $(P<0.05)$.

Oxidative stress (OS) is defined as an imbalance between production of free radicals and reactive metabolites, which are called reactive oxygen species (ROS), and ROS elimination by protective mechanisms which are referred to as antioxidants, and this imbalance leads to damage of important molecules and cells, with potential impact on the whole organism [20]. In addition, cancer initiation and progression have been shown to be associated with oxidative stress by increasing DNA mutations or inducing DNA damage, genome instability, and cell proliferation [21]. Previous studies showed that an increased rate of oxidative stress plays an important role in the pathogenesis of N-SCLC $[8,9]$. In the current study, our results suggest that the levels of MDA and $\mathrm{NO}$ were higher in the patient group than that in the control group, but the level of T-AOC was lower in the patient group than that in the control group.

Glutathione S-transferases (GSTs), an important super family of phase II drug-metabolizing enzymes that respond to oxidative stress, include at least seven distinct classes, namely, $\alpha(\mathrm{A}), \mu(\mathrm{M}), \pi(\mathrm{P}), \sigma($ Sigma $), \zeta$ (Zeta), $\omega$ (Omega) and $\theta(\mathrm{T})$, and play an important role in cell protection by catalyzing the conjugation of a large variety

Table 3 Genetic polymorphisms of glutathione S-transferase (GST) M1 and T1 in relation to plasma MDA, NO and T-AOC

\begin{tabular}{|c|c|c|c|c|c|c|c|c|c|c|c|c|c|}
\hline Group & $\mathrm{N}$ & \multicolumn{4}{|l|}{ MDA (nmo/lmL) } & \multicolumn{4}{|l|}{ NO (nmol/mL) } & \multicolumn{4}{|l|}{ T-AOC (units/mL) } \\
\hline Control & 100 & \multicolumn{4}{|l|}{$8.49 \pm 3.30$} & \multicolumn{4}{|l|}{$16.79 \pm 5.37$} & \multicolumn{4}{|l|}{$13.69 \pm 4.56$} \\
\hline Case & 110 & \multicolumn{4}{|l|}{$11.67 \pm 4.31$} & \multicolumn{4}{|l|}{$19.68 \pm 4.11$} & \multicolumn{4}{|l|}{$9.88 \pm 3.58$} \\
\hline$P$ & & \multicolumn{4}{|l|}{$<0.001$} & \multicolumn{4}{|l|}{$<0.001$} & \multicolumn{4}{|l|}{$<0.001$} \\
\hline \multicolumn{14}{|l|}{ GSTM1 } \\
\hline$(+)$ & 44 & \multicolumn{4}{|l|}{$10.18 \pm 4.23$} & \multicolumn{4}{|l|}{$18.01 \pm 3.56$} & \multicolumn{4}{|l|}{$11.11 \pm 2.67$} \\
\hline$(-)$ & 66 & \multicolumn{4}{|l|}{$12.67 \pm 4.09$} & \multicolumn{4}{|l|}{$20.80 \pm 4.09$} & \multicolumn{4}{|l|}{$9.07 \pm 3.88$} \\
\hline P & & \multicolumn{4}{|l|}{$<0.001^{a} ; 0.003^{b}$} & \multicolumn{4}{|l|}{$<0.001^{a} ;<0.001^{b}$} & \multicolumn{4}{|l|}{$<0.001^{a} ; 0.001^{b}$} \\
\hline \multicolumn{14}{|l|}{ GSTT1 } \\
\hline$(+)$ & 35 & \multicolumn{4}{|l|}{$9.29 \pm 2.84$} & \multicolumn{4}{|l|}{$17.98 \pm 3.99$} & \multicolumn{4}{|l|}{$11.73 \pm 2.82$} \\
\hline$(-)$ & 75 & \multicolumn{4}{|l|}{$12.78 \pm 4.44$} & \multicolumn{4}{|l|}{$20.48 \pm 3.94$} & \multicolumn{4}{|l|}{$9.03 \pm 3.58$} \\
\hline P & & \multicolumn{4}{|l|}{$<0.001^{a} ;<0.001^{c}$} & \multicolumn{4}{|l|}{$<0.001^{a} ; 0.003^{c}$} & \multicolumn{4}{|l|}{$<0.001^{a} ;<0.001^{c}$} \\
\hline GSTM1/T1 & & Control & Case & & $P$ & Control & Case & & $P$ & Control & Case & & $P$ \\
\hline$(+/+)$ & & $23 \quad 7.81 \pm 2.97$ & 20 & $8.56 \pm 2.86$ & 0.047 & $23 \quad 15.74 \pm 4.38$ & 20 & $17.68 \pm 3.57$ & 0.049 & $23 \quad 14.71 \pm 4.29$ & 23 & $11.97 \pm 2.28$ & 0.046 \\
\hline$(+/-)$ & & $35 \quad 8.45 \pm 3.01$ & 24 & $11.52 \pm 4.76$ & 0.018 & $35 \quad 16.05 \pm 5.13$ & 24 & $18.28 \pm 3.60$ & 0.032 & $35 \quad 13.68 \pm 4.79$ & 35 & $10.39 \pm 2.80$ & 0.028 \\
\hline$(-/+)$ & & $24 \quad 8.47 \pm 2.98$ & 15 & $10.27 \pm 2.60$ & 0.021 & $24 \quad 16.23 \pm 4.90$ & 15 & $18.37 \pm 4.59$ & 0.027 & $24 \quad 13.59 \pm 4.92$ & 24 & $11.40 \pm 3.47$ & 0.024 \\
\hline$(-/-)$ & & $18 \quad 8.81 \pm 3.12$ & 51 & $13.38 \pm 4.20$ & 0.008 & $18 \quad 18.02 \pm 4.29$ & 51 & $21.51 \pm 3.68$ & 0.004 & $18 \quad 10.27 \pm 5.01$ & 18 & $8.39 \pm 3.75$ & 0.003 \\
\hline P & & & $<0.0$ & $01^{a} ;<0.001^{d}$ & & & $<0.0$ & $01^{\mathrm{a}} ;<0.001^{\mathrm{d}}$ & & & $<0.0$ & $01^{a} ;<0.001^{d}$ & \\
\hline
\end{tabular}

+, present genotype; -, null genotype; MDA, malondialdehyde; NO, nitric oxide; T-AOC, total antioxidant capacity.

${ }^{a}$ compared with control.

${ }^{\mathrm{b}}$ compared with GSTM1 (+).

'compared with GSTT1 (+).

d compared with GSTM1/T1 (+/+). 
of endogenous and exogenous compounds, including ROS and carcinogenic compounds and their metabolites. Human cytosolic GST genes exhibit genetic polymorphisms, and many genetic polymorphisms lead to altered GST activities, which may be partially responsible for the individual host's susceptibility to oxidative damage. Previous studies suggested that GSTM1 and GSTT1 null genotypes may be associated with increased susceptibility to lung cancer $[15,16]$, but other studies have shown no associations between GSTM1 and T1 null genotypes for lung cancer risk $[17,18]$. In our results, we found that the frequencies of the GSTM1, T1, and M1/T1 null genotypes were significantly higher in the patient group than that in the control group. Moreover, we found that OR for GSTM1 (null/present) in T1 null genotype $(\mathrm{OR}=4,132)$ and present genotype $(\mathrm{OR}=0,719)$ groups, and $\mathrm{OR}$ for GSTT1 (null/present) in M1 null genotype $(\mathrm{OR}=4.533)$ and present genotype $(\mathrm{OR}=1.826)$ groups are heterogeneous. It was shown that a significant susceptibility to oxidative damage is not attributable to the presence of either the M1 or T1 gene. Both genes must be positive for the significance of OR.

Furthermore, the association between the GSTM1 and T1 genotypes and level of oxidative stress in the patient group was analyzed in the current study. We found that the activities of MDA and NO in the GSTM1, T1 and M1/T1 null genotype groups were statistically significantly higher than that in the GSTM1, T1 and M1/T1 present genotype groups. The level of $\mathrm{T}$-AOC in the GSTM1, GSTT1 and GSTM1/T1 null genotype groups were statistically significantly lower than that in the GSTM1, T1 and M1/T1 present genotype groups. Moreover, we found that for one of the genes of M1 and T1 to be positive is not sufficient for susceptibility to oxidative damages. Both genes must be positive for an important increase in susceptibility to oxidative damages.

\section{Conclusions}

Our results suggest that oxidative damage may play an important role in patients with N-SCLC and that the GSTM1 and GSTT1 null genotypes may predispose the tissues of patients with N-SCLC to increased oxidative damage. Both null genotypes must be positive for an important increase in the susceptibility to oxidative damages. Therefore, more attention should be paid to oxidative stress-related pathological manifestations in patients with N-SCLC who bear the GSTM1 and GSTT1 null genotypes. Furthermore, studies on glutathione S-transferases gene polymorphisms and levels of oxidative stress should be done in a multicenter, multi-ethnic population and with a large number of patients with N-SCLC in the future.

\section{Abbreviations}

GSTM1: glutathione S-transferase M1; GSTT1: glutathione S-transferase T1; N-SCLC: non-small lung cancer; PCR: multiplex polymerase chain reaction;
OS: oxidative stress; MDA: malondialdehyde; NO: nitric oxide; T-AOC: total antioxidant capacity.

\section{Competing interests}

The authors declare that they have no competing interests.

\section{Authors' contributions}

$\mathrm{KT}$ designed the research; $\mathrm{HZ}, \mathrm{XW}, \mathrm{YX}, \mathrm{MC}, \mathrm{ZL}$ and $\mathrm{XW}$ performed the research; and $\mathrm{HZ}$ analyzed the data and drafted the manuscript. All authors read and approved the final version of the manuscript.

\section{Acknowledgements}

We are grateful to all subjects who participated in this study. This project was supported by the Application Fundamentals Foundation of Yunnan Province, China (Grant No. 2010ZC198) and National Natural Science Fund of China (Grant No. 81300541).

\section{Author details}

${ }^{1}$ Affiliated Yan'an Hospital of Kunming Medical University, No. 245 Renmin East Road, Kunming 650051, China. ${ }^{2}$ Affiliated Hospital of Guiyang Medical College, No. 9 Beijing Road, Guiyang 550004, China.

Received: 29 January 2014 Accepted: 17 November 2014

Published online: 04 December 2014

\section{References}

1. Thun MJ, Hannan LM, Adams-Campbell LL, Boffetta P, Buring JE, Feskanich D, Flanders WD, Jee SH, Katanoda K, Kolonel LN, Lee IM, Marugame T, Palmer JR, Riboli E, Sobue T, Avila-Tang E, Wilkens LR, Samet JM: Lung cancer occurrence in never-smokers: an analysis of 13 cohorts and 22 cancer registry studies. PLoS Med 2008, 5:e185.

2. Jemal A, Siegel R, Xu J, Ward E: Cancer statistics, 2010. CA Cancer J Clin 2010, 60:277-300.

3. Wu W, Platoshyn O, Firth AL, Yuan JX: Hypoxia divergently regulates production of reactive oxygen species in human pulmonary and coronary artery smooth muscle cells. Am J Physiol Lung Cell Mol Physiol 2007, 293:L952-959.

4. Kinnula VL, Crapo JD: Superoxide dismutases in the lung and human lung diseases. Am J Respir Crit Care Med 2003, 167:1600-1619.

5. Farinati F, Piciocchi M, Lavezzo E, Bortolami M, Cardin R: Oxidative stress and inducible nitric oxide synthase induction in carcinogenesis. Dig Dis 2010, 28:579-584.

6. Cadet J, Douki T, Ravanat JL: Oxidatively generated base damage to cellular DNA. Free Radic Biol Med 2010, 49:9-21.

7. Hair JM, Terzoudi GI, Hatzi VI, Lehockey KA, Srivastava D, Wang W, Pantelias GE, Georgakilas AG: BRCA1 role in the mitigation of radiotoxicity and chromosomal instability through repair of clustered DNA lesions. Chem Biol Interact 2010, 188:350-358.

8. Ito K, Yano T, Morodomi Y, Yoshida T, Kohno M, Haro A, Shikada Y, Okamoto T, Maruyama R, Maehara Y: Serum Antioxidant Capacity and Oxidative Injury to Pulmonary DNA in Never-smokers with Primary Lung Cancer. Anticancer Res 2012, 32:1063-1067.

9. Peddireddy V, Badabagni SP, Gundimeda SD, Penagaluru PR, Mundluru HP: Assessment of 8-oxo-7, 8-dihydro-2'-deoxyguanosine and malondialdehyde levels as oxidative stress markers and antioxidant status in non-small cell lung cancer. Biomarkers 2012, 17:261-268.

10. Strange RC, Spiteri MA, Ramachandran S, Fryer AA: Glutathione-S-transferase family of enzymes. Mutat Res 2001, 482:21-26.

11. Nebert DW, Vasiliou V: Analysis of the glutathione S-transferase (GST) gene family. Hum Genomics 2004, 1:460-464.

12. Seidegard J, Ekstrom G: The role of human glutathione transferases and epoxide hydrolases in the metabolism of xenobiotics. Environ Health Perspect 1997, 105(Suppl 4):791-799.

13. Eaton DL, Bammler TK: Concise review of the glutathione S-transferases and their significance to toxicology. Toxicol Sci 1999, 49:156-164.

14. Pemble S, Schroeder KR, Spencer SR, Meyer DJ, Hallier E, Bolt HM, Ketterer B, Taylor JB: Human glutathione S-transferase theta (GST1): CDNA cloning and the characterization of a genetic polymorphism. Biochem J 1994, 300(Pt 1):271-276. 
15. Pinarbasi H, Silig Y, Cetinkaya O, Seyfikli Z, Pinarbasi E: Strong association between the GSTM1-null genotype and lung cancer in a Turkish population. Cancer Genet Cytogenet 2003, 146:125-129.

16. Matakova T, Sivonova M, Halasova E, Mistuna D, Dzian A, Berzinec P, Letkova L, Dobrota D: Gene polymorphisms of biotransforming enzymes (GSTs) and their association with lung cancer in the Slovakian population. Eur J Med Res 2009, 14(Suppl 4):275-279.

17. Tamaki Y, Arai T, Sugimura H, Sasaki T, Honda M, Muroi Y, Matsubara Y, Kanno S, Ishikawa M, Hirasawa N, Hiratsuka M: Association between cancer risk and drug-metabolizing enzyme gene (CYP2A6, CYP2A13, CYP4B1, SULT1A1, GSTM1, and GSTT1) polymorphisms in cases of lung cancer in Japan. Drug Metab Pharmacokinet 2011, 26:516-522.

18. To-Figueras J, Gene M, Gomez-Catalan J, Galan MC, Fuentes M, Ramon JM, Rodamilans M, Huguet E, Corbella J: Glutathione S-transferase M1 (GSTM1) and T1 (GSTT1) polymorphisms and lung cancer risk among Northwestern Mediterraneans. Carcinogenesis 1997, 18:1529-1533.

19. Tang K, Xue W, Xing Y, Xu S, Wu Q, Liu R, Wang X, Xing J: Genetic polymorphisms of glutathione s-transferase $\mathrm{M1}, \mathrm{T} 1$, and $\mathrm{P} 1$, and the assessment of oxidative damage in infertile men with varicoceles from northwestern china. J Androl 2012, 33:257-263.

20. Durackova Z: Some current insights into oxidative stress. Physiol Res 2010, 59:459-469.

21. Visconti R, Grieco D: New insights on oxidative stress in cancer. Curr Opin Drug Discov Devel 2009, 12:240-245.

doi:10.1186/s40001-014-0067-3

Cite this article as: Zhang et al: Genetic polymorphisms of glutathione $\mathrm{S}$-transferase $\mathrm{M} 1$ and $\mathrm{T} 1$, and evaluation of oxidative stress in patients with non-small cell lung cancer. European Journal of Medical Research 2014 19:67.

\section{Submit your next manuscript to BioMed Central and take full advantage of:}

- Convenient online submission

- Thorough peer review

- No space constraints or color figure charges

- Immediate publication on acceptance

- Inclusion in PubMed, CAS, Scopus and Google Scholar

- Research which is freely available for redistribution 